\title{
The Role of Jankovic Spasm Grading, Orbicularis Oculi Muscle Function and Functional Improvement Scale Pre-and Post-Treatment in Dosing Botulinum Toxin A in Treatment of Essential Blepharospasm, Meige's Syndrome and Hemifacial Spasm
}

\section{Bastola $\mathbf{P}^{1 *}$ and Koirala $\mathbf{S}^{2}$}

${ }^{1}$ Associate Professor, Consultant Ophthalmologist and Oculoplastic Surgeon, Botulinum Toxin A Expert, Kathmandu, Nepal

${ }^{2}$ Head of Department, Department of Neurology, DM Neurologist, National

Academy of Medical Sciences, Kathmandu, Nepal

*Corresponding Author: Bastola P, Associate Professor, Consultant Ophthalmologist

and Oculoplastic Surgeon, Botulinum Toxin A Expert, Kathmandu, Nepal.

DOI: 10.31080/ASOP.2020.03.0144
Received: June 12, 2020

Published: July 10, 2020

(C) All rights are reserved by Bastola $\mathbf{P}$ and

Koirala S.

\begin{abstract}
Background: Botulinum Toxin A (BTX A) is a proven medication used in neurological disorders like Meige's syndrome (MS), essential blepharospasm (ES) and hemifacial spasm (HS). Jankovic spasm grading is a time trusted grading system to detect the severity of these movement disorders pre-treatment. Orbicularis oculi muscle weakness and functional improvement ratings post treatment are trusted guidelines to judge the efficacy of BTX A.

Aim/Objective: The study aimed to find out the correct dosing of BTX A in treating cases of MS, ES and HS using Jankovic Spasm grading pre-treatment and orbicularis oculi muscle weakness and functional impairment improvement post treatment.

Methods: This was a hospital based, interventional, prospective study. All diagnosed consecutive patients of HS, ES and MS attending the neuro-Ophthalmologic/oculoplastic clinic, general outpatient department of Ophthalmology and or referred diagnosed cases were enrolled for the study, an informed consent was taken from all the patients before the treatment for medico-legal issues. The pre-treatment dosing was done using Jankovic spasm grading, post treatment assessment was done using orbicularis oculi muscle assessment and functional improvement. The study subjects were followed up to 9 months or till spasms reappeared.

Results: A total of 50 study subjects were enrolled in the study, $80 \%$ of them were women. The highest number of study subjects were in ES (50\%) group followed by HS (40\%) and MS (10\%). Mean Jankovic spasm grading in all study subjects was 3.59 ( \pm 0.38 Std. deviation). The study groups ES and MS required a higher dose of BTX A (P value: < 0.0001) when compared with HS study group. Mean orbicularis oculi muscle weakening and functional impairment improvement scale in all study subjects was 2.7 ( \pm 0.3 Std. deviation) and $3.8( \pm 0.6)$ respectively. Mean duration of action till the spasms reappeared in the study subjects was 5.1 months $( \pm 1.7$ Std. deviation). HS and ES study groups had better treatment when compared to MS.

Conclusions: Jankovic spasm grading pre-treatment and orbicularis oculi muscle weakness grading and functional improvement score post treatment plays a very effective role to titrate the correct dose of BTX A in patients of HS, ES and MS.
\end{abstract}

Keywords: Botulinum Toxin A; Essential Blepharospasm; Hemifacial Spasm; Jankovic Spasm Grading; Meige’s Syndrome

Citation: Bastola P and Koirala S. "The Role of Jankovic Spasm Grading, Orbicularis Oculi Muscle Function and Functional Improvement Scale Pre-and Post-Treatment in Dosing Botulinum Toxin A in Treatment of Essential Blepharospasm, Meige's Syndrome and Hemifacial Spasm”. Acta Scientific Ophthalmology 3.8 (2020): 06-12. 


\section{Introduction}

The movement disorders of face and neck regions are mainly essential blepharospasm (ES), hemifacial spasm (HS) and Meige's syndrome (MS). The first record of blepharospasm and lower facial spasm was found in the 16th century in a painting titled De Gaper. At that time, and for several ensuing centuries, patients with such spasms were regarded as being mentally unstable and often were institutionalized in insane asylums. Little progress was made in the diagnosis or treatment of blepharospasm until the early 20th century, when Henry Meige (pronounced "mehzh"), a French neurologist, described a patient with eyelid and midface spasms, spasm facial median, a disorder now known as Meige's syndrome [1].

Hemifacial spasm (HS) represents a segmental myoclonus of muscles innervated by the facial nerve and was first described by Gowers in the year 1884 [2].

The cause remains obscure, treatment less than ideal, and frustration is a major factor as it is with blepharospasm. Many physicians, even regarding the more common ES, may have had no experience with MS and the patient may be told the process is psychological. Movement disorders such as ES, HS and MS are treated by different modalities, including surgery $[3,4]$.

Botulinum toxin (BTX) is a neurotoxic protein produced by the bacterium Clostridium botulinum and related species. It prevents the release of the neurotransmitter acetylcholine from axon endings at the neuromuscular junction and thus, causes flaccid paralysis. Infection with the bacterium causes the disease botulism. Types A and B can cause disease in humans and are also used commercially and medically [5].

Botulinum toxin is the most poisonous substance known. Intoxication can occur naturally because of either wound or intestinal infection or by ingesting preformed toxin in food. The estimated human lethal dose of type A toxin is $1.3-2.1 \mathrm{ng} / \mathrm{kg}$ intravenously or intramuscularly, 10 - $13 \mathrm{ng} / \mathrm{kg}$ when inhaled, or $1000 \mathrm{ng} / \mathrm{kg}$ when taken by mouth. Commercial forms are marketed under various brands. Botulinum toxin is used to treat many disorders characterized by overactive muscle movement including spasms of the head and neck, eyelid, vagina, limbs, jaw, and vocal cords and cosmetic purpose, migraine, strabismus and excessive sweating etc. to mention few [5-7].
Botulinum toxin A; injection is now widely accepted treatment modality for ES, HS, and MS and is in practice for temporary relief of symptoms in head and neck movement disorders like ES, MS and HS [5]. BTX A known for its potency, when used in ES, HS and MS with correct dosing can be very effective, this study with the back ground of movement disorders of head and neck region and the potency of BTX A aimed at finding out the correct dosing system by correlating the effect of BTX A with patients with various grades of Jankovic spasm and relief post treatment. This study also is intended to provide some literature regarding correct dosing of BTX A and various gradings to be used specially in countries; where the existing literature is not much.

\section{Methodology}

This was a hospital based interventional; descriptive study carried out over a decade in various eye hospitals and private clinics of Nepal. All the consecutive patients diagnosed with essential blepharospasm, Meige's syndrome or hemifacial spasm at neuroOphthalmology clinic, oculoplasty clinic, outpatient department of Ophthalmology or referred cases were included in the study. All patients underwent full clinical evaluation, including neurological as well as ocular examinations. An informed consent was taken from all subjects. The study followed the declaration of Helsinki. The subjects also gave consent to take videos and photographs during and post treatment during the study. All the subjects were provided an information sheet about treatment with BTX A and its potential complications.

Jankovic spasm grading [8] was used as a standard pre-treatment assessment tool to calculate BTX A dose as given below:

- Grade: 0 - Normal,

- Grade 1-Slight disability, no functional impairment,

- Grade 2 - Moderate disability, no functional impairment,

- $\quad$ Grade 3 - Moderate disability, functional impairment and

- Grade 4 - Incapacitated.

Two observer's observed the spasm and graded it as given above. Cohen's Kappa (k) value of 1 was taken as complete agreement between the observers.

Citation: Bastola P and Koirala S. "The Role of Jankovic Spasm Grading, Orbicularis Oculi Muscle Function and Functional Improvement Scale Pre-and Post-Treatment in Dosing Botulinum Toxin A in Treatment of Essential Blepharospasm, Meige's Syndrome and Hemifacial Spasm”. Acta Scientific Ophthalmology 3.8 (2020): 06-12. 
The Role of Jankovic Spasm Grading, Orbicularis Oculi Muscle Function and Functional Improvement Scale Pre-and Post-Treatment in Dosing Botulinum Toxin A in Treatment of Essential Blepharospasm, Meige's Syndrome and Hemifacial Spasm

Botox, Allergan, Inc. was used for treatment in all cases. The manufacturer's instructions were carefully followed. The toxin was used within 4 hours of its reconstitution and was kept in fridge for cooling purpose. A vial of 100 units freeze dried BTX A was reconstituted with $4 \mathrm{ml}$ of preservative free $0.9 \%$ saline solution to yield toxin in a concentration of 2.5 units per $0.1 \mathrm{ml}$. The toxin was injected subcutaneously into the selected sites using a $1 \mathrm{ml}$ tuberculin syringe with a 27 - 291/2-gauge needle. Atropine, adrenaline injections were kept standby during the treatment process with antiallergics to combat any complications. The initial dose was calculated based on clinical judgement of the spasm and after observing the subjects video for few minutes. Five to seven periocular injections in ES/MS were selected with each site getting 2.5 - 7.5 units of BTX A depending upon the severity of spasm of the affected muscle. In hemifacial spasm five periocular sites as above were selected but only in one side of the face. The cheek was injected in cases having a severe degree of retraction of angle of mouth. Patients were evaluated at two weeks, six weeks and six months and 9 months' time or when the spasm reappeared. The subjects were advised to maintain a diary to note the onset of clinical response, peak effect, duration of clinical improvement and complications on follow up assessment. Telephone contact with the patients was encouraged for follow up assessment and through designated social media. Post treatment the patients were evaluated for the following after detailed ocular examination.

Assessment of functional improvement $[7,8]$ was done following a grading system given below:

- 0 - no effect,

- 1 - Mild effect but no functional improvement

- 2 - Moderate improvement but no change in functional disability

- 3 - Moderate change in both severity and function

- 4 - Marked improvement in severity and function in a similar way.

Orbicularis oculi muscle weakening [9] grading tool was done by assessing the closure of the eyes:
- Grade 0: Incomplete eyelid closure,

- Grade 1: Lids just closing, minimal resistance to overcome

- Grade 2: Closing well, some resistance, easily overcome

- Grade 3: Strong closure, can be overcome with difficulty

- Grade 4: Very strong closure, cannot be overcome or overcome with extreme difficulty.

Assessment of functional impairment rating and grading of orbicularis oculi muscle weakening post injections was done by two different observers. Cohen's Kappa ( $\mathrm{k}$ ) value of 1 was taken as complete agreement between the observers.

Grade 0, 1, 2 were considered as good outcome post treatment with BTX A

Complications were looked for and noted specially blepharoptosis, deviation of angle of mouth, bruises and ecchymosis. Patients were followed up at six weeks and six months and nine months and similar examinations were carried out as mentioned in the two weeks visit (disappearance of complications if present were also noted). Patients were told to note the date and follow up immediately as soon as significant appearance of spasms occurred. A proforma was maintained to record all the regarding patient, treatment and follow up visits. Useful data was entered in the database for statistical analysis SPSS 20, Stata were used for statistical analysis. A statistician was consulted when and where felt necessary.

\section{Results}

Fifty subjects were enrolled for the study, 50\% (25 subjects) were diagnosed with ES, followed by $40 \%$ (20 subjects) with HS and $10 \%$ with MS. $80 \%$ (40) of them were females and the $20 \%$ (10 subjects) males. Mean age of the female and male subjects was 52 $( \pm 10$, range $29-73)$ and $54( \pm 9.8$, range: $45-70)$ years. Dry eyes, stress, watching television, talking, lack of sleep were prominent exacerbating factors. Pre-treatment Jankovic spasm grading for calculation of dose of BTX A in different sub groups and the impact of BTX A in the study group with regard to orbicularis oculi muscle function, functional impairment improvement scale and reappearance of the spasms have been described in table 1 and 2 . 
The Role of Jankovic Spasm Grading, Orbicularis Oculi Muscle Function and Functional Improvement Scale Pre-and Post-Treatment in Dosing Botulinum Toxin A in Treatment of Essential Blepharospasm, Meige's Syndrome and Hemifacial Spasm

Jankovic spasm (JS) grading in study subjects pre-treatment

\begin{tabular}{|c|c|c|c|c|c|}
\hline \multicolumn{6}{|c|}{ Jankovic spasm (JS) grading in study subjects pre-treatment } \\
\hline Diagnosis & Number of subjects & JS grading (Minimum) & JS grading (Maximum) & \multicolumn{2}{|c|}{ Mean (SD**) } \\
\hline ES & 25 & 3 & 4 & \multicolumn{2}{|c|}{$3.6( \pm 0.5)$} \\
\hline MS† & 5 & - & 4 & \multicolumn{2}{|c|}{4} \\
\hline HS* & 20 & 2 & 4 & \multicolumn{2}{|c|}{$3.18( \pm 0.66)$} \\
\hline Total & 50 & - & - & \multicolumn{2}{|c|}{$3.59( \pm 0.38)$} \\
\hline \multicolumn{6}{|c|}{ Botulinum toxin A (BTX A) treatment and dose in units for each study sub groups } \\
\hline \multirow[t]{2}{*}{ BTX A Dose } & \multicolumn{3}{|c|}{ Diagnosed sub groups } & & \\
\hline & ES & MS & HS & Total (\%) & $P$ value \\
\hline 20 & & & & & \multirow{10}{*}{$<0.0001$} \\
\hline 23 & & & $3(15 \%)$ & $3(6 \%)$ & \\
\hline 25 & & & $12(60 \%)$ & $12(24 \%)$ & \\
\hline 30 & & & $5(25 \%)$ & $5(10 \%)$ & \\
\hline 35 & $1(4 \%)$ & & & $1(2 \%)$ & \\
\hline \multicolumn{5}{|l|}{40} & \\
\hline 45 & $5(20 \%)$ & & & $5(10 \%)$ & \\
\hline 50 & $10(40 \%)$ & & & $10(20 \%)$ & \\
\hline 60 & $9(36 \%)$ & $5(100 \%)$ & & $9(18 \%)$ & \\
\hline Total & $25(100 \%)$ & $5(100 \%)$ & $20(100 \%)$ & $50(100 \%)$ & \\
\hline
\end{tabular}

Table 1: Pre-treatment Jankovic spasm grading and calculation of dose of botulinum toxin A based on the grading.

*Hemifacial spasm, † Meige’s syndrome, ‡ Essential blepharospasm, **Standard deviation.

Table 1 showing that the mean JS grading was higher for ES and MS and BTX A dose was also given higher for the same study groups.

$P$ value was significant when different sub-groups were compared to BTX A dose, subjects in ES and MS needing significantly higher dose of BTX A when compared to HS.

\begin{tabular}{|c|c|c|c|c|}
\hline \multicolumn{5}{|c|}{ Orbicularis oculi muscle weakening grading in study sub groups in two weeks follow up } \\
\hline Diagnosis & Number of subjects & Grading (Minimum) & Grading (Maximum) & Mean (SD*) \\
\hline ES & 25 & 1 & 3 & $2( \pm 0.2)$ \\
\hline MS & 5 & 1 & 2 & $1.5( \pm 0.3)$ \\
\hline HS & 20 & 1 & 3 & $2.7( \pm 0.3)$ \\
\hline Total & 50 & 1 & & $3.3)$ \\
\hline \multicolumn{5}{|c|}{ Functional impairment improvement scale in study sub groups in first follow up } \\
\hline Diagnosis & Number of subjects & Grading (Minimum) & Grading (Maximum) & Mean (SD*) \\
\hline ES & 25 & 2 & 4 & $3.5( \pm 0.7)$ \\
\hline MS & 5 & 2 & 4 & $3.2( \pm 1.0)$ \\
\hline HS & 20 & 3 & 3.8 & $3.8( \pm 0.1)$ \\
\hline Total & 50 & 2 & $0.6)$ \\
\hline \multicolumn{5}{|c|}{ The noted time of spasm reappearance by the study subjects in different treatment sub-groups } \\
\hline Diagnosis & Study subjects & Minimum (Months) & Maximum (Months) & Mean (SD*) \\
\hline ES & 25 & 2.5 & 6.5 & $5( \pm 1.4)$ \\
\hline MS & 5 & 2.5 & 6.5 & $4.5( \pm 2.4)$ \\
\hline HFS & 20 & 3.5 & 8.5 & $6.0( \pm 1.3)$ \\
\hline Total & 50 & 2.5 & 8.5 & $5.1( \pm 1.7)$ \\
\hline
\end{tabular}

Table 2: Orbicularis oculi muscle weakening post treatment with BTX A, functional impairment improvement scale and reappearance of spasms in the study sub groups.

*Standard deviation.

Table 2 showing the study sub groups ES and HS having comparatively better outcome with the treatment than MS.

Citation: Bastola P and Koirala S. "The Role of Jankovic Spasm Grading, Orbicularis Oculi Muscle Function and Functional Improvement Scale Pre-and Post-Treatment in Dosing Botulinum Toxin A in Treatment of Essential Blepharospasm, Meige's Syndrome and Hemifacial Spasm”. Acta Scientific Ophthalmology 3.8 (2020): 06-12. 


\section{Discussion}

Amongst 50 subjects enrolled in the study the study had $80 \%$ of women gender wise, the mean age of study subjects in the study was 52 and 54 years respectively for women and men. Common exacerbating factors in study subjects were dry eyes, stress, lack of sleep etc. These findings of the study correlated well with the existing knowledge about essential blepharospasm, hemifacial spasm and Meige's syndrome and other studies done elsewhere [8-10].

Current study had $50 \%$ cases of essential blepharospasm followed very closely by $40 \%$ cases of HS, which did not correlate with some other reported prevalence of ES, HS and MS. The reported prevalence for HS is highest followed by ES and MS $[8,10,11]$ this may be contributed to the small number of study population and various hospitals for treatment and also probably contributed by the fact that subjects who had severe symptoms or were unable to carry out their day to day work attended the clinic more.

Mean Jankovic spasm grading in the study subjects was $3.6( \pm$ $0.5), 4( \pm 0)$ and $3.18( \pm 0.66)$ in ES, MS and HS respectively, while the mean combined of all subjects was $3.59( \pm 0.66)$, which highlighted the fact that study subjects came for treatment when their movement disorder and spasms were severe enough to disturb their daily routine work and in MS group using Jankovic spasm grading all cases were incapacitated. This was very important in dosing BTX A for treatment of these subjects. Study subjects having high Jankovic spasm grading (Table 1) were given more BTX A, whereas subjects with lower Jankovic spasm grading were given comparatively lower dose of BTX A injection and in lesser sites (Table 1).

In the current study both pre-tarsal as well as pre-septal injections of BTX A was given, and the treatment outcome was better (Table 2). This finding again correlated with studies done elsewhere; where the results of injecting BTX A also in pretarsal portion of orbicularis oculi muscle was more effective [12,13].

The study subjects in ES and MS required more dosage of BTX A than HS, this can be explained by the following; the study subjects in the former groups had higher mean Jankovic spasm grading (Table 1) and also due to the fact that hemifacial spasms subjects had only one side of the face involved. This finding correlated well with studies done in the past $[2,8]$.
In our study, the mean (Standard deviation) orbicularis oculi weakening in first follow up for ES, MS and HS was $2( \pm 0.2), 1.5( \pm$ $0.3)$ and $1.5(0.3)$ respectively, with an overall mean of $2.7( \pm 0.3)$ for all the study subjects. This was an excellent result and showed that the dosing for BTX A was correct and correlated very well with Jankovic spasm grading pre-treatment (Table 1 and 2).

Similarly, in the current study the mean functional impairment improvement in ES, MS and HS study sub groups was $3.5( \pm 0.7)$, $3.2( \pm 1.0)$ and $3.9( \pm 0.6)$ with an overall mean of $3.8( \pm 0.6)$. This finding again highlighted how important is it to correctly grade Jankovic spasms and calculate the dose (Table 1 and 2). An interesting finding in the study was the mean improvement for MS was lesser than ES and HS it is primarily due to the fact that the study subjects in MS group were only five and also, they all were incapacitated when graded and hence were given maximum possible dose at their first presentation. Hemifacial spasm group had the best outcome with both orbicularis oculi weakening and functional improvement rating, which correlated with studies done in the past $[4,7,8,14]$.

The mean reappearance of spasm in months in all study subjects in the current study was 5.1 months $( \pm 1.7)$. However, HS study sub group had an excellent temporary relief with the treatment lasting up to 8.5 months, closely followed by essential blepharospasm, this study sub group had a mean of 5 months $( \pm 1.4)$ and many study participants reported the spasms after 8 months. MS study group reappearance correlated well with orbicularis oculi weakening grading and functional improvement rating, a smaller sample size and initial cautious approach could be the reasons for temporary relief lasting up to 2.5 - 6.5 months (Table 2).

These findings in the study again strongly rationalized the fact that initial Jankovic spasm grading is very important to get the maximum benefit from correct or near correct dose of botulinum toxin A. These findings again correlated with studies done elsewhere $[4,7,8]$.

Minor complications like deviation of angle of mouth, transient ptosis and bruise or ecchymosis were seen in three study subjects during injection or in first follow up, the subjects recovered from them pretty well in due course of time, this finding correlated with studies done in the past [15-17]. 
The Role of Jankovic Spasm Grading, Orbicularis Oculi Muscle Function and Functional Improvement Scale Pre-and Post-Treatment in Dosing Botulinum Toxin A in Treatment of Essential Blepharospasm, Meige's Syndrome and Hemifacial Spasm

\section{Recommendations}

Jankovic spasm grading plays a very important role in dosing botulinum toxin A to treat movement disorders of head and neck region specifically Meige's syndrome, essential blepharospasm and hemifacial spasm. However, we recommend that post treatment assessment of orbicularis oculi muscle and functional improvement must assessed to know whether the given dose of BTX A was near correct low or high, so as to verify and give correct dose when the subject follows up with spasms again.

\section{Limitations}

The prevalence of movement disorders is very low so the study sample size was small. This study findings may not be same if a larger study done in titrating the dose of botulinum toxin $\mathrm{A}$.

\section{Conclusion}

Botulinum toxin A is a very safe, effective temporary medical treatment modality to treat movement disorders like essential blepharospasm, Meige's syndrome and hemifacial spasm. Botulinum toxin A when administered with correct dosing following the grading systems used in the study can provide a temporary relief up to eight months or more. The results of treatment are very promising with near correct dose, we recommend it for a wider use in other parts of Nepal and world.

\section{Acknowledgements}

We would like to thank all the study subjects who unconditionally accepted to be part of the research and did not leave the study in between. We would also like to thank Prof. JP Agrawal, Prof. DN Shah who initiated idea of using botulinum toxin A in Nepal and supported the researcher to register it in Nepal for therapeutic use. We would like to thank Mrs. Sheela Koirala Bastola for keeping all the study subjects records intact and also for entering the data in SPSS and Stata.

\section{Conflict of Interest}

None.

\section{Source of Funding}

None.

\section{Bibliography}

1. Houser M and Waltz T. "Meige syndrome and pallidal deep brain stimulation". Movement of Disorder 20.9 (2005): 12031205.
2. Abbruzzese G., et al. "Hemifacial spasm". Handbook of Clinical Neurology 100 (2011): 675-680.

3. Jankovic J. "Etiology and differential diagnosis of blepharospasm and oromandibular dystonia". Advances in Neurology 49 (1988): 103-116.

4. Tan NC., et al. "Hemifacial spasm and involuntary facial movements”. QJ Medicine 95 (2002): 493-500.

5. Joseph Jankovic and Mitchell F Brin. "Botulinum toxin: Historical perspective and potential new indications". Muscle Nerve 20 (1997): 129-145.

6. Hallett Mark. "One Man's Poison. Clinical Applications of Botulinum Toxin". The New England Journal of Medicine 341 (1999): 118-120.

7. Thussu A., et al. "Botulinum toxin treatment of hemifacial spasm and blepharospasm: objective response evaluation". Neurology India 47 (1999): 206-209.

8. Bastola P., et al. "The role of the injection Botulinum Toxin A in cases of Essential Blepharospasm Syndrome, Hemifacial Spasm and Meige's Syndrome". Kathmandu University Medical Journal 8.3 (2001): 305-310.

9. Rahman R., et al. "A new grading system for assessing orbicularis muscle function". Eye 17 (2003): 610-612.

10. Gulevich Steven. eMedicine. Hemifacial Spasm (2005).

11. Donald CF. "Essential blepharospasm". In: Yanoff M, Duker Jay S.CD- ROM Ophthalmology: Mosby International (1998).

12. Tan A. "Botulinum Toxin for Neurological Disorders in a Movement Disorders Clinic in Singapore". Singapore Medical Journal 39 (1998): 403-405.

13. Cakmur R., et al. "Comparison of preseptal and pretarsal injections of botulinum toxin in the treatment of blepharospasm and hemifacial spasm". Journal of Neurology 249 (2002): 6468.

14. Bastola P. "Orbicularis oculi muscle function and functional improvement rating as grading tools to evaluate the efficacy of Botulinum Toxin A in patients with movement disorders of head and neck". Bangladesh Ophthalmology Journal 2 (2016): 11-18.

15. Price J and O'Day J. "Efficacy and side effects of botulinum toxin treatment for blepharospasm and hemifacial spasm". Australian and New Zealand Journal of Ophthalmology 4.22 (1994): 255-260. 
16. Maurri S., et al. "Beneficial effect of botulinum A toxin in blepharospasm: 16 months' experience with 16 cases". The Italian Journal of Neurological Sciences 9 (1988): 337-344.

17. Snir M., et al. "Quantitative changes in botulinum toxin A treatment over time in patients with essential blepharospasm and idiopathic hemifacial spasm". American Journal of Ophthalmology 136 (2003): 99-105.

\section{Assets from publication with us}

- Prompt Acknowledgement after receiving the article

- Thorough Double blinded peer review

- Rapid Publication

- Issue of Publication Certificate

- High visibility of your Published work

Website: www.actascientific.com/

Submit Article: www.actascientific.com/submission.php

Email us: editor@actascientific.com

Contact us: +919182824667

Citation: Bastola P and Koirala S. "The Role of Jankovic Spasm Grading, Orbicularis Oculi Muscle Function and Functional Improvement Scale Pre-and Post-Treatment in Dosing Botulinum Toxin A in Treatment of Essential Blepharospasm, Meige's Syndrome and Hemifacial Spasm”. Acta Scientific 